\title{
Primary Umbilical Endometriosis: A Case Report
}

Endometriosis (EM) is defined as extrauterine functional endometrial tissue, commonly found in the pelvic peritoneum, ovaries, or rectovaginal septum. This is a chronic condition that affects $6-15 \%$ women of reproductive age and $6 \%$ of postmenopausal women (1). The most common symptoms are dysmenorrhea and dyspareunia (1), although 20-25\% patients are asymptomatic (1-3). Infertility affects 30$50 \%$ of patients (2).

Umbilical endometriosis, Villar's nodule, or spontaneous umbilical endometrioma are terms for a rare condition with an estimated prevalence of $0.5 \%$ to $1.0 \%$ among all endometriosis locations. It was described by Villar in 1886 and is defined as the presence of endometrial tissue within the umbilicus without prior history of pelvic endometriosis (4). There is no consensus on the exact genesis. Several theories are thought to be possible causes of the development of endometriosis, including retrograde menstruation, direct spread, embryonal rest, coelomic metaplasia, and lymphatic or hematogenous spreading (5). The most common symptom of primary umbilical endometriosis (PUE) is a palpable mass in the umbilical region. Secondary umbilical endometriosis is explained by iatrogenic implantation of ectopic endometrial cells after surgery, usually in surgical scars after cesarean section, laparoscopic surgery, and episiotomy (5). Furthermore, there is a hypothesis that the umbilicus may act as a physiological scar and a seeding site (6).

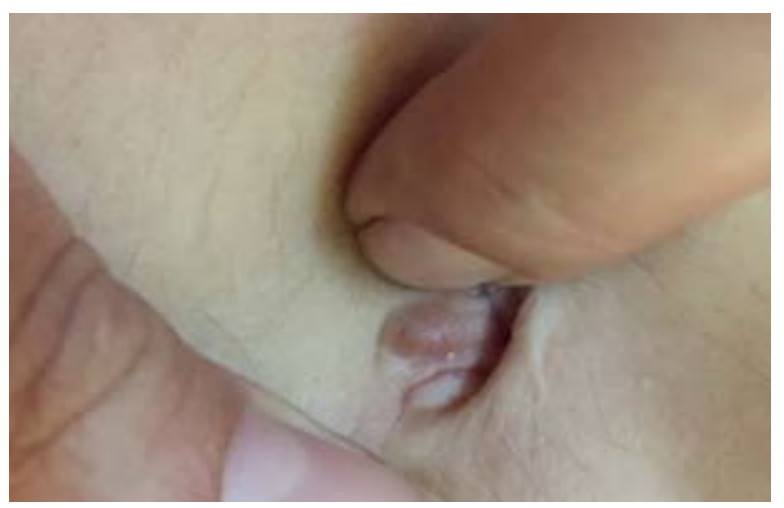

Figure 1. Noduli umbilici
Our patient initially presented with an asymptomatic clinical picture following periumbilical pain after presentation of the first local recurrence, while navel bleeding appeared in the second recurrence during period. It is unknown whether she actually had recurrences or if surgery failed to remove it all at the first place.

A nulliparous female patient aged 39 was referred to our Clinic due to a palpable mass in her umbilicus. No other symptoms were reported. A firm and indurated mass in the center of the umbilicus about $2 \mathrm{~cm}$ in diameter was found by physical examination (Figure 1). Dermoscopy revealed small red globular structures ("red atolls") within regularly distributed homogeneous reddish pigmentation fading at the periphery. The dermoscopic features had the same characteristics as the first dermoscopic findings of umbilical endometriosis reported by Di Giorgi et al. in 2003, where the author proposed dermoscopic findings in the diagnosis (Figure 2). Ultrasonography revealed an indeterminate solid lesion in the subcutis at the umbilicus presenting as a hypoechoic mass with indistinct margins, $25 \times 15 \mathrm{~cm}$ in diameter, extending within the whole depth of the subcutaneous adipose tissue and providing minimal Doppler signal. The gynecological report was without pathological findings. The patient had never undergone surgical procedures or had trauma at the site of the lesion. Her periods were regular, and she had no dysmenorrhea.

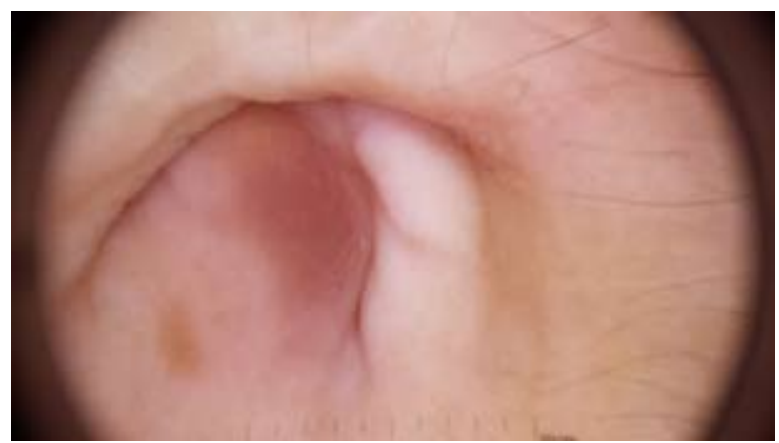

Figure 2. Dermoscopy: Small red globular structures (,,red atolls") within regularly distributed homogeneous reddish pigmentation 
Family history was unremarkable. Excision of the lesion was performed, and histopathological findings revealed endometriosis of cutaneous and subcutaneous adipose tissue (Figure 3, Figure 4). Two and a half months later, after a recurrence of the disease was found upon regular check up by ultrasound examination, the patient underwent another extirpation of the lesion. Two years after following the last pathological findings, the patient once again reported to our clinic, only this time complaining of bleeding through the umbilicus during her last period. Another recurrence of the primary disease was found and removed under local anesthesia.

Umbilical endometriosis is rare, especially in patients with no previous history of pelvic surgery or preexisting pelvic (primary) endometriosis. Mean age of presentation is $37.7 \pm 0.98$ years (4). It is typically presented by a firm, pigmented, or bluish nodule with pain and tenderness associated with cyclic bleeding or discharge during menstruation.

The pathogenesis of spontaneous cutaneous endometriosis is unknown. The most commonly accepted theories are coelomic metaplasia, direct extension through the round ligament or the patent omphalomesenteric duct, congenital presence of developmentally-displaced endometrial tissue, or mechanical seeding of endometrial tissue via the lymphatic or venous system (7). The "retrograde menstruation theory" proposes the spread of endometrial tissue by retrograde menstruation and implantation of those cells into the pelvic structures (8). As a contribution to theories mentioned above, there it is possible that the umbilicus acts as a physiological scar with a predilection for endometrial tissue (4). The differential diagnosis includes benign nevus, umbilical polyp, endosalpingitis presented as periumbilical papules, pyogenic/foreign body granuloma, seborrheic keratosis, epithelial inclu-

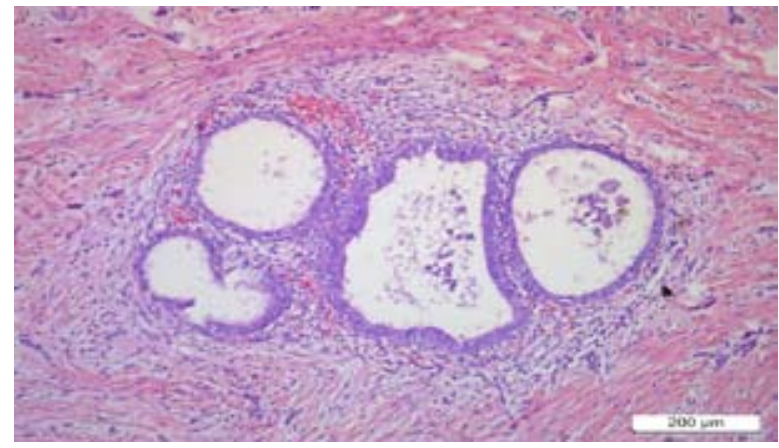

Figure 3. Magnification $40 \times 10$, hematoxyline-eosine staining. Focus of endometrial glandular epithelium and stroma within the skin connective and subcutaneous tissue. sion cyst, lipoma, umbilical hernia, desmoid tumor, hemangioma, granular cell tumor, omphalitis, keloid sebaceous cyst, and urachus anomaly. Primary neoplasms or metastatic deposits from an intra abdominal malignancy, termed Sister Mary Joseph's nodule, should also be ruled out (7-9).

Even though ultrasonography showed no pathognomonic signs of PUE, it appeared that both a CT scan and MRI proved to be of a little value in diagnosing intra-abdominal endometriosis. Only ultrasonography could provide information on the size of the nodule and its adherence to surrounding tissues (10). Histopathologic analyses are still the mainstay for diagnosis of PUE (4).

The treatment of choice for Villar's nodule is surgical treatment. Medical therapy is rarely curative (6) and includes progesterone, norethisterone, danazol, and gonadotropin-releasing hormone analogues. Some authors reported improvement in reducing nodule size and improvement in symptoms using medical hormonal therapies (9).

Surgical choices include either total umbilical resection with or without repair of the surrounding fascia and peritoneum, or local excision of the endometrial nodule while preserving the umbilicus. For some authors, local excision of endometrial lesions with negative margins is still the treatment of choice since recurrences appear to be rare, while others prefer total resection of the umbilicus (9).

Cutaneous endometriosis should be taken under consideration in all unclear lesions and in the presence of umbilical discharge or bleeding. A unique aspect of our case is that the patient developed clear and conclusive symptoms after the second recurrence. The utility of total excision of the umbilicus in these in order to prevent recurrences cases remains to be further investigated.

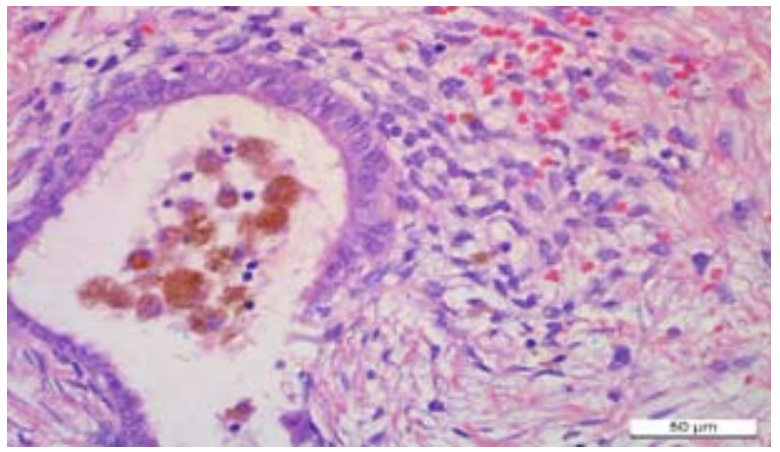

Figure 4. Magnification $40 \times 11$, hematoxyline-eosine staining. Focus of endometrial tissue with fagocytosed hemosiderin in macrophage (remnants of previous bleedings) 


\section{References:}

1. Boesgaard-Kjer Daniel, Boesgaard-Kjer Diana, Kjer Jorgen J. Primary umbilical endometriosis (PUE). Ejogrb. 2017;209:44-5.

2. Bulletti C, Coccia ME, Battistoni S, Borini A. Endometriosis and infertility. J Assist Reprod Genet. 2010;27:441-7.

3. Victory R, Diamond MP, Johns DA. Villars nodule: a case report and systematic literature review of endometriosis externa of the umbilicus. J Minim Invasive Gynecol. 2007;14:23-32.

4. Sengupta M, Naskar A, Gon S, Majumdar B. Villar's nodule. Online J Health Allied Scs. 2011;10:19.

5. Taniguchi F, Hirakawa E, Azuma Y, UejimaCh, Ashida K, Harada T. Primary umbilical endometriosis: unusual and rare clinical presentation-case report. Case Rep Obstet Gynecol. 2016;2016:9302376.

6. Dave AM, Dave AA, Margolin DJ. A Case of Umbilical Endometriosis: Villar's Nodule. Cureus Dec 2016;8:e926.

7. Agarwal A, Fong ZF. Cutaneous endometriosis. Singapore Med J. 2008;49:704-9.

8. Van den Nouland DPA, Kaur M. Primary umbilical endometriosis: a case report. Fact Views Vis Obgyn. 2017;9:115-9.

9. Egin S, Pektas BA, Hot S, Mihmanli V. Primary umbilical endometriosis: A painful swelling in the umbilicus concomitantly with menstruation. Int J Surg Case Rep. 2016;28:78-80.

10. Panicker SCR, Pillai N, Nagarsekar U. Villar's Nodule. A rare presentation of external endometriosiscase report. MJAFI. 2010;66:70-1.

\section{Olivera Levakov, Branislava Gajić, Zorica Gajinov, Milana Ivkov-Simić, Ljuba Vujanović, Zoran Golušin}

Dermatovenerology Department, Clinical Center of Vojvodina, Faculty of Medicine University Novi Sad

\section{Corresponding author:}

Olivera Levakov, MD

Faculty of Medicine, University of Novi Sad

Clinic of Dermatovenereology Diseases

Clinical Center of Vojvodina

Hajduk Veljkova Street 1

21137 Novi Sad

Serbia

olivera.levakov@mf.uns.ac.rs

Received: November 30, 2019

Accepted: November 16, 2020 